\title{
On Maximizing Lifetime of a Sensor Cluster
}

\author{
Samar Agnihotri, Pavan Nuggehalli, H. S. Jamadagni \\ Centre for Electronics Design and Technology, Indian Institute of Science, Bangalore - 560012, India \\ Email: \{samar, pavan, hsjam\}@ cedt.iisc.ernet.in
}

\begin{abstract}
We consider the energy consumed in radio transmission of a set of sensors forming a data gathering wireless network. Our objective is to enhance the lifetime of such networks by exploiting three system-level opportunities. Firstly, the number of bits to be transmitted can be reduced by taking advantage of the redundancy induced by spatio-temporal correlation in sensor data. Secondly, channel coding allows us to reduce transmission energy at the cost of increased transmission time. Thirdly, sensor nodes can be expected to operate collaboratively, allowing optimal management of distributed energy resources. Our main contribution lies in providing a framework to merge these ideas for energy conscious networking. We pose the problem of maximizing network lifetime as an optimal scheduling problem. We first consider a special case where data rate is linearly proportional to received signal power. In this scenario, we investigate both static and $d y$ namic scheduling strategies. The optimal static schedule turns out to have a very simple form. For the dynamic case, we obtain an integer linear program formulation to find the optimal strategy. We then propose an efficient algorithm that exploits the special nature of the problem setting to quickly find the optimal solution. Finally, we consider the general case where data rates and signal power need not be linearly related and propose an algorithm to find the optimal transmission times subject to the deadline constraint imposed by the system.
\end{abstract}

\section{Introduction}

This paper considers the problem of maximizing the lifetime of a data gathering wireless sensor network in which a set of sensors periodically sample a field and transmit the data to a central location or base station. It is expected that these sensor nodes will run on tiny non-renewable batteries and in many instances be placed in inaccessible locations, making it impossible to replace dead nodes. In such a scenario, a key challenge is to devise system-level strategies to optimally utilize finite energy resources. Sensor nodes consume energy in sensing/actuating, computation and communication. Advances in device technology and low-power electronics have greatly reduced the energy cost of sensing and computation. However, the energy cost of communication is determined by the harsh nature of wireless links and has the potential to be a significant bottleneck. We define network lifetime to be the time until the first node runs out of energy. This is a popular definition $[1,2]$ and is particularly appropriate when a single node failure can be disastrous (for example, reducing sensor coverage or causing network partition). We note that other definitions of network lifetime used in literature include fraction of surviving nodes in a network [3] and mean expiration time [4]. In this paper, we are interested in managing the energy expended by nodes for communication purposes, with the objective of maximizing the operating lifetime of sensor networks. We limit ourselves to the case where nodes communicate to the base station in a single hop. This model, apart from being useful for small networks, is also applicable to sensor clusters within a large network [5].

With this simple network model, we exploit three available opportunities to meet the challenge of enhancing network lifetime. Firstly, the number of bits to be transmitted can be reduced by taking advantage of the redundancy induced by spatio-temporal correlation in sensor data. In [6], Slepian and Wolf show that it is possible to compress a set of correlated sources down to their joint entropy, without explicit communication between the sources. Practical techniques [7] based on distributed Slepian-Wolf source coding have also been proposed recently. Another possibility of exploiting source redundancy opens up when the base-station is not energy constrained. In this case, the base station can assume most of the burden of communication [8] and the need for sophisticated source coding disappears. Secondly, channel coding [9] allows us to reduce the energy required for transmission at the cost of increased transmission time. Finally, sensor nodes are cooperative allowing optimal management of distributed energy resources. Our main contribution lies in providing a framework to merge these ideas to control the energy cost of radio transmission 
to maximize network lifetime.

There is much related work in this area. Cooperation among sensor nodes has been exploited mainly via energy efficient routing $[1,10,11,12,13,14]$. Our study was motivated by previous research in $[16,15]$ which explicitly incorporate aggregation costs in gathering sensor data. In [15], the authors consider the problem of correlated data gathering by a network with a sink node and a tree communication structure. Their goal is to minimize the total transmission (energy) cost of transporting information. The first part of [16] considers a model similar to our, namely, that of several correlated nodes transmitting directly to a base station. However, both $[15,16]$ are interested in minimizing total energy expenditure, as opposed to maximizing network lifetime. In the latter case, the optimal solution is shown in both papers to be a greedy solution based on ordering sensors according to their distance (which reflects data aggregation cost) from the base station. However, we show that this solution is not optimal for maximizing network lifetime. Moreover, to the best of our knowledge, a thorough investigation of joint source-channel coding for maximizing lifetime is lacking and this paper is an attempt in that direction.

In section 2, we present our system model and describe our notion of instantaneous decoding. In section 3, we consider a scenario that allows us to neglect the impact of transmission time allocation. This is similar to the scenario considered given in $[15,16]$. We present the optimal static scheduling strategy under instantaneous decoding. The collaborative nature of sensor networks is then exploited by considering dynamic strategies, which are shown to improve lifetime. We also provide a fast algorithm to determine dynamic strategies. In section 4 , we consider a general channel model which allows us to consider the joint impact of source and channel coding on system lifetime. We provide some insight for this problem and an algorithm for finding the optimal transmission times. Numerical and simulation results are provided in section 5 .

\section{System Model}

We consider a network of $N$ battery equipped sensor nodes strewn uniformly in a coverage area. Initially, sensor node $k, 1 \leq k \leq N$, has access to $E_{k}$ units of energy. The wireless channel between sensor $k$ and the base station is described by a path loss factor $d_{k}$ that captures various channel effects such as distance induced attenuation, shadowing, and multipath fading. For simplicity, we assume $d_{k}$ 's to be constant. This is reasonable for static networks and can also be extended to scenarios where the path loss parameter varies slowly and can be accurately tracked.

The network operates in a time-division multiple access (TDMA) mode. In each slot, every sensor takes samples of the coverage area and in its allotted time, communicates its data to the base station. A discrete random process $X_{k}^{n}$ represents the sampled sensor reading at node $k$ in the $n^{t h}$ time slot. The entropy of $X_{k}^{n}$ is denoted by $H\left(X_{k}\right)$. We assume that sensor readings in any time slot are spatially correlated. We ignore temporal correlation by assuming that sensor readings in different time slots are independent. Temporal correlation can be easily incorporated in our work for data sources satisfying the Asymptotic Equipartition Property (AEP). The general problem is to find the optimal rate (the number of bits to transmit) and transmission times for each node, which maximize network lifetime. Both the rate and time allocation are constrained. The rate allocation should fall within the Slepian-Wolf achievable rate region and the sum of transmission times should be less than the period of a time-slot (which is taken to be unity). Finding the optimal rate allocation is a computationally challenging problem as the Slepian-Wolf achievable rate region for $N$ nodes is defined by $2^{N}-1$ constraints. We simplify the problem by insisting that decoding at the base-station be instantaneous in the sense that once a particular node has been polled, the data generated at that node is recovered at the base-station before the next node is polled. This reduces the rate allocation problem to finding the optimal scheduling order, albeit at some loss of optimality. Let $\Pi$ be the set of permutations of the set, $\{1,2, \ldots, N\}$. The polling schedule followed by the network in any time slot corresponds to a permutation, $\pi \in \Pi$. Let $\pi(k)$ denote the $k^{\text {th }}$ node to be scheduled. Instantaneous decoding implies that the amount of data to be transmitted by node $\pi(k)$ is the conditional entropy of the data source at node $\pi(k)$, given the data generated by all previously polled nodes. We denote the amount of information generated by node $\pi(k)$ by $H_{k}^{\pi}$. Our aim is to find the scheduling strategy (scheduling order and transmission time allocation) that maximizes network lifetime.

\section{Small Rate Region Approximation}

In this section, we assume that transmission rate is linearly proportional to signal power. This assumption is motivated by Shannon's AWGN capacity formula which is approximately linear for low data rates. The energy expended by a node to transmit $H$ units of information is given by $H \times d$, where $d$ is the suitably normalized path loss factor between the node and the base station. The linear rate assumption implies that transmit energy is independent of transmission time. Hence, the optimal time allocation problem is trivial and we only need to find the optimal scheduling order. We consider two kinds of schedules, namely, static and dynamic. In static scheduling, the nodes follow the same fixed scheduling order in all time slots until the network dies. Under dynamic scheduling, we allow nodes to collaborate further by allowing them to employ different 
schedules in different time slots.

\subsection{Static scheduling}

In static scheduling, each permutation, $\pi \in \Pi$ corresponds to a TDMA schedule. The number of bits transmitted by node $\pi(k)$ (under instantaneous decoding) is given by $H\left(X_{\pi(k)} \mid X_{\pi(k-1)}, \ldots, X_{\pi(1)}\right)$. The optimal scheduling order is given by the solution to the following max-min optimization problem.

$$
\max _{\pi \in \Pi} \min _{1 \leq k \leq N} \frac{E_{\pi(k)}}{d_{\pi(k)} H\left(X_{\pi(k)} \mid X_{\pi(k-1)}, \ldots, X_{\pi(1)}\right)}
$$

The objective function represents the lifetime of node $\pi(k)$ under the given static schedule $\pi$. We describe next a scheduling strategy, Minimum Cost Next (MCN), which turns out to be an optimal static scheduling strategy. When a scheduling decision needs to be taken, MCN chooses that node (among the unpolled ones) which will consume the least amount of energy. The algorithm is given as follows. The MCN schedule is denoted by $\pi$.

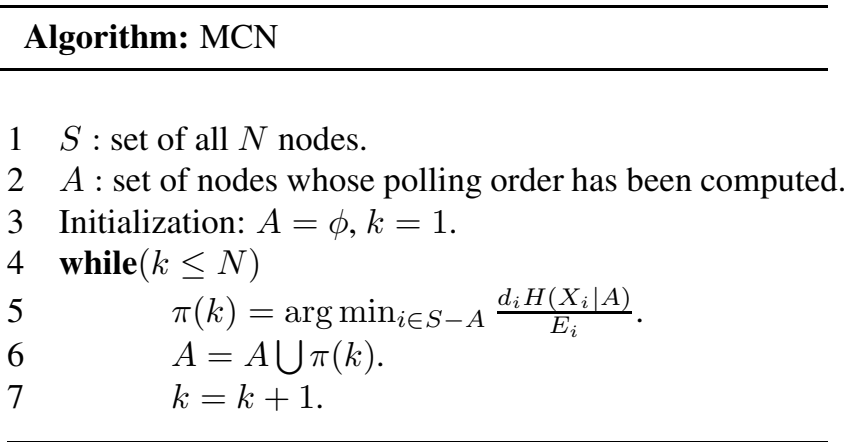

Theorem 1 The Minimum Cost Next schedule is an optimal static schedule.

Proof: Omitted for brevity.

\subsection{Dynamic scheduling}

In this section, we explore how network lifetime can be increased by employing multiple schedules. Instead of restricting the network to follow a single schedule, we allow the system to employ different schedules over time. There are $N$ ! possible schedules to choose from. Let $H_{k}^{i}$ be the number of information bits generated per slot by node $k$ under the $i^{t h}$ schedule, $1 \leq k \leq N, 1 \leq i \leq N$ !. The optimal lifetime $L$, in this case is found as follows

$$
\begin{gathered}
L=\max \sum_{i=1}^{N !} \tau_{i} \\
\text { s.t. } \sum_{i=1}^{N !} d_{k} H_{k}^{i} \tau_{i} \leq E_{k}
\end{gathered}
$$

$\tau_{i}$ is the number of slots for which the $i^{\text {th }}$ schedule is used. The constraints ensure that the time assignment is feasible for each node with respect to its energy capability. Note that (2) in an integer linear program problem. However, for most sensor applications, slot durations will be a small fraction of network lifetime. So, it is reasonable to model $\tau_{i}$ 's as real numbers rather than integers and to treat (2) as a linear program. A dynamic schedule, $\tau$, is given by the set $\left\{\tau_{i}\right\}$. A static schedule can also be thought as a dynamic schedule where only one of the $\tau_{i}$ 's is non-zero.

Given $N$ ! variables, in general, there seems to be no easy way to solve (2) to compute the optimal $\tau_{i}$ values. However, the special nature of our problem can be exploited to yield efficient methods. It is reasonable to assume that sensor readings at a node are strongly correlated only with neighboring nodes. For any schedule, the energy consumed by a node will then depend primarily on its relative order in the schedule with respect to its neighbors. This clustering phenomena leads to considerable reduction in computational effort. Moreover, the max-min nature of our optimization problem simplifies the search for an optimal schedule and allows us to easily determine when a schedule is optimal. Both these properties are exploited in our algorithm which we refer to as Lifetime Optimal Clustering ALgorithm (LOCAL) and describe as follows.

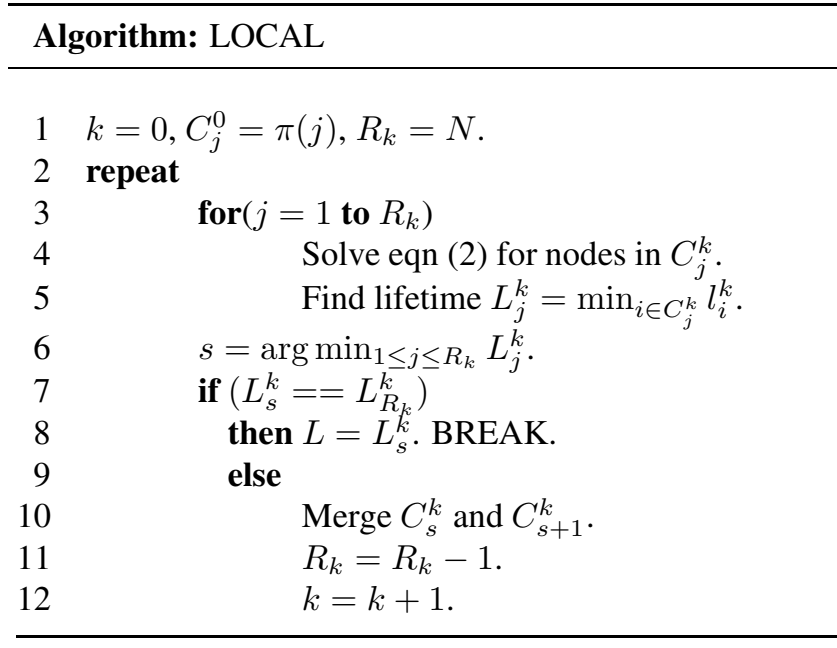

Starting with the MCN schedule defined earlier, LOCAL proceeds in stages by successively yielding better schedules till no further improvement in lifetime is possible. Initially each node forms its own cluster. Let $R_{k}$ be the number of clusters in stage $k$ and $C_{j}^{k}, 1 \leq j \leq R_{k}$ denote the $j^{\text {th }}$ cluster. The lifetime $L_{j}^{k}$ of $C_{j}^{k}$, under any dynamic schedule is the minimum of the lifetimes of the nodes in $C_{j}^{k}$. Given a set of clusters, LOCAL considers only those schedules which maintain the clustering order. In other words, all nodes in cluster $C_{j}^{k}$ will be scheduled before any node in the clusters, $C_{l}^{k}, \quad j+1 \leq l \leq R_{k}$. Within a cluster however, nodes are dynamically scheduled to maximize 
cluster lifetime. This reduces the problem of scheduling $N$ nodes in (2) to scheduling only the nodes within a cluster, decreasing computational complexity. At each stage then, the lifetime of each node is calculated using (2) for intracluster scheduling. Cluster formation in LOCAL follows a greedy approach inspired by the max-min definition of network lifetime. At the beginning of stage $k$, consider the lifetimes of the clusters formed in stage $k-1$ under the dynamic schedule $\tau^{k-1}$. Let cluster $C_{i}^{k-1}$ have the minimum lifetime among all clusters. If there are more than one such cluster, we choose the cluster with the lowest index. If the lifetime of the last cluster, $C_{R_{k-1}}^{k-1}$, is equal to the minimum lifetime, then the algorithm terminates. If not, merge the nodes in cluster $C_{i}^{k-1}$ and cluster $C_{i+1}^{k-1}$ to form a new cluster in stage $k$ and repeat the process. Note that in the worst case, LOCAL will reduce to (2) if all the nodes merge into one single cluster. Our simulations results show that such an event is very unlikely even for fairly large networks.

A further optimization is achieved by exploiting the max-min nature of our problem as follows. Suppose that, after stage $k$, the cluster lifetimes are such that for some $l$

$$
\min _{1 \leq j \leq l} L_{j}^{k} \geq \max _{l+1 \leq j \leq R_{k}} L_{j}^{k}
$$

Note that network lifetime can never be improved by scheduling nodes in the first $l$ clusters after other nodes in the network. Hence we can ignore the scheduling of nodes in the first $l$ clusters and focus only on the remaining nodes.

\section{Lemma 1 LOCAL is optimal.}

Proof: Omitted for brevity.

\section{General case}

So far, we have assumed that transmission rate is linearly proportional to received signal power. However, in general, this assumption is not valid. For example, by inverting Shannon's channel capacity formula for the AWGN channel, it is straight forward to show [9] that transmission energy is a strictly decreasing convex function of transmission time. Other channel coding situations lead to a similar result. In such a scenario, not only do we have to find the optimal (static or dynamic) scheduling order but also the optimum transmission times for each node. Let $f(h, x)$ be the energy required to transmit $h$ bits of information in $x$ units of time with unit path loss. Based on our discussion, we model the energy function $f(h, x)$ as follows.

1. $f(h, x)$ is a strictly decreasing continuous positive function in $x$.

2. $\lim _{x \rightarrow 0} f(h, x)=\infty$
As before, we denote by $H_{k}^{i}$ the number of information bits generated per slot by node $k \in[1 \ldots N]$ under the $i^{t h}$ schedule, $1 \leq i \leq N$ !. Let $\omega_{k}^{i}$ be the corresponding transmission time alloted to node $k$. The optimal static schedule is the solution to the following optimization problem.

$$
\begin{array}{r}
\max _{1 \leq i \leq N !} L^{i} \\
\text { s.t. } \quad L^{i} f\left(H_{k}^{i}, \omega_{k}^{i}\right) d_{k} \leq E_{k} \\
\\
\sum_{k=1}^{N} \omega_{k}^{i} \leq 1, \quad \omega_{k}^{i} \geq 0
\end{array}
$$

Here $L^{i}$ is the lifetime achievable by the system under the $i^{\text {th }}$ schedule. Note that lifetime is integer-valued but as in section 3, we will continue to treat it as a real number.

The solution to the optimal scheduling problem for the general case turns out to be very different from the solution considered in section 3. Firstly, nodes can collaborate with each other to a much greater extent than before by varying their transmission times. For example, nearby nodes can finish their transmissions sooner allowing far away nodes more time to transmit in order to improve system lifetime. Secondly the optimal scheduling order is dependent on the correlation structure as well as the energy function and greedy algorithms such as MCN are, in general, not optimal. Our initial research appears to suggest that the problem of finding the optimal schedule is non-trivial and may even be NP-hard. Since our model is more applicable to sensor clusters rather than sensor networks themselves, a brute force approach to find the optimal scheduling algorithm may well suffice. The challenge then is to find the optimal allocation of transmission times, given a particular scheduling order. The key to finding the optimal transmission times lies in the following lemma.

Lemma 2 Given a schedule, there exists a unique set of transmission times $\left\{\omega_{k}\right\}$ which maximizes network lifetime. Moreover, a set of transmission times is optimal if and only if $\sum_{k=1}^{N} \omega_{k}=1$ and all the nodes consume the same amount of energy.

Proof: Omitted for brevity.

Lemma 2 implies that, in the optimal scenario, all nodes run out of energy at the same time. We leverage lemma 2 to devise an algorithm to find the optimal transmission times. We call this algorithm the Channel Aware algorithm (CA). The CA algorithm proceeds as follows. Assume $L_{0}$ is a feasible value of network lifetime and $H_{k}$ is the number of bits which node $k$ needs to transmit. Let $\delta$ be an arbitrarily chosen positive step value.

In the CA algorithm, network lifetime is increased by a constant step-size $\delta$, till the deadline constraint ( $T$ should be less than 1) is violated. Then, the step size $\delta$, is decreased exponentially till a feasible value of lifetime is 
found. Clearly, this algorithm will eventually converge. From lemma 2, it follows that the lifetime value obtained by CA is optimal. The quantity $\epsilon$ determines the speed of convergence.

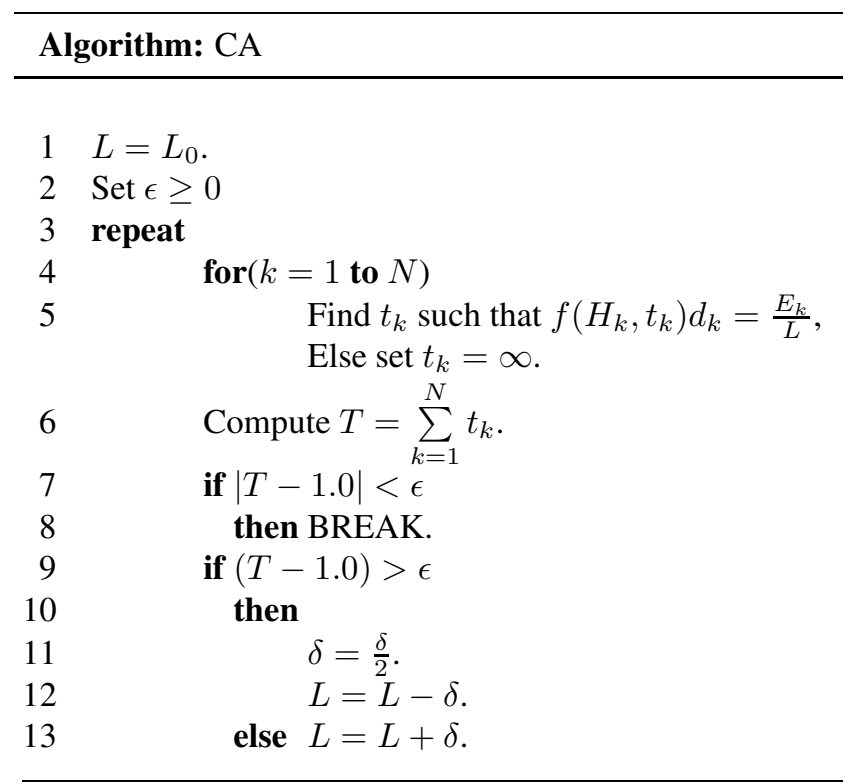

At this stage, it is natural to ask if dynamic scheduling can further improve performance. We have seen experimentally that such is indeed the case though the improvement is not dramatic and we do not report the results here.

\section{Results}

Our sensor network consists of $N$ nodes uniformly distributed in a unit circle, with the base station at the center. Each node starts out with 10,000 units of energy. Further, we model the data generated at the sensors by an $\mathrm{N}$ dimensional Gaussian vector with zero mean and covariance matrix $K$. We assume that the correlation between two sources is distance dependent, so we define the covariance between nodes $i$ and $j$ as $K_{i j}=\sigma e^{-\alpha d_{i j}^{2}}$.

In Fig. 1a, we plot the average network lifetime achieved by the MCN and LOCAL algorithms for different values of node density and compare their performances with the performance of a reference scheme that does not exploit the spatial-correlation among sensor readings. For each node density value, 500 scenarios $^{1}$ are used to calculate the average network lifetime. As expected, LOCAL performs consistently better than MCN. Fig. 1b, compares the execution time of LOCAL with the time taken to solve the linear program in (2), denoted BigLP. LOCAL can not only run 10 to 100 times faster than BigLP, but also handle larger networks.Fig. 2 compares the $\mathrm{CA}$ algorithm with a reference

\footnotetext{
${ }^{1}$ Increasing the number of scenarios beyond 500 did not provide qualitatively different results.
}

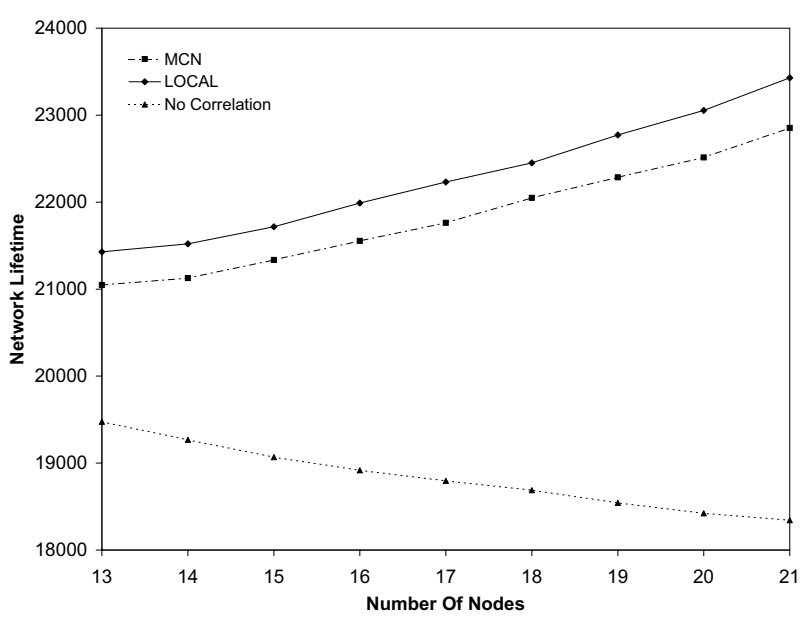

(a)

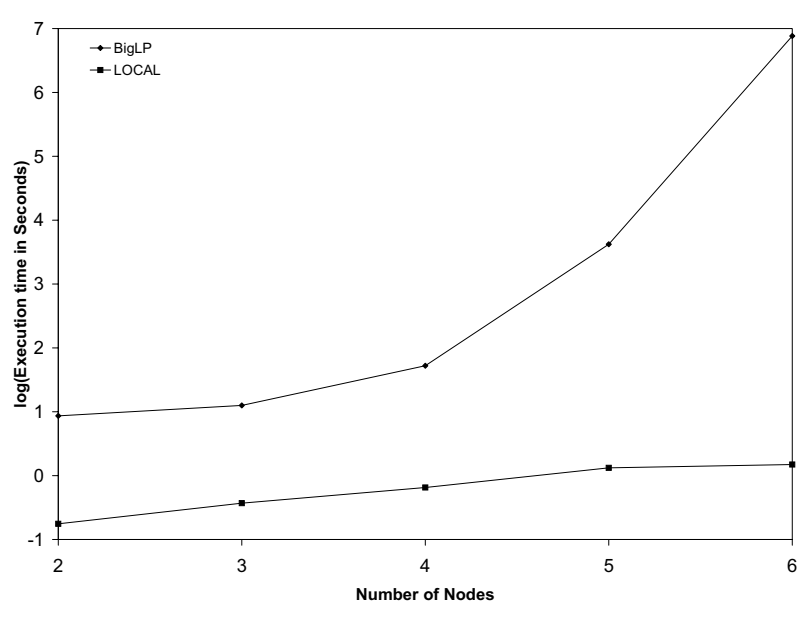

(b)

Figure 1. (a) Average network lifetime comparison between MCN, LOCAL, and the reference algorithm. (b) Execution times comparison between BigLP and LOCAL.

scheme that allocates equal transmission times to all nodes. Both the schemes follow the schedule where the nodes are polled in the order of their proximity to the base station.

\section{Conclusions and Future work}

This paper considered the problem of maximizing the lifetime of a data gathering wireless network. Our contribution differs from previous research in two respects. Firstly, we proposed a joint source-channel coding framework to mitigate the energy cost of radio transmission. Secondly, 


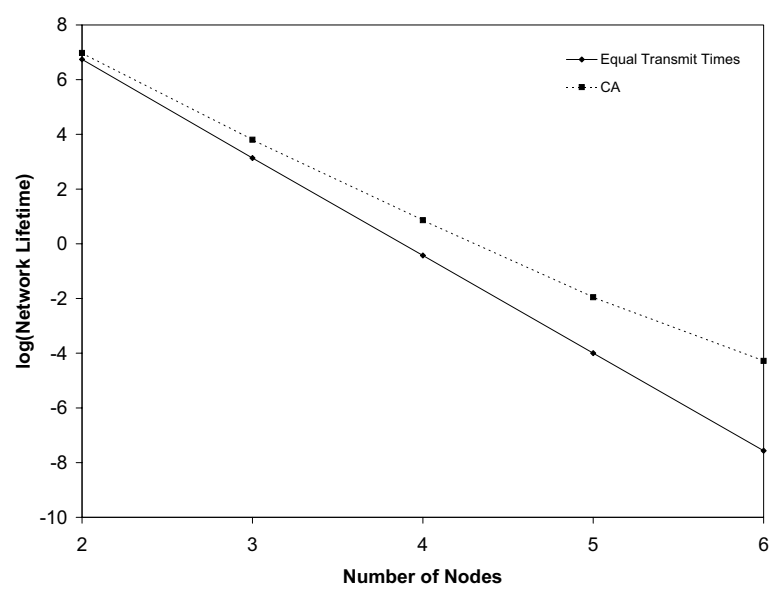

Figure 2. Comparison of the Channel Aware (CA) algorithm with the reference scheme.

we have explicitly maximized network lifetime as opposed to other objective functions such as cumulative energy cost. In our system model, nodes communicate directly to a base station in a time division multiplexed manner. With our notion of instantaneous decoding, we show that the network lifetime maximization problem reduces to finding an optimal scheduling strategy (polling order and transmission time allocation).

Much work remains to be done. A thorough exploration of the computation complexity of the problems posed in the paper is required. In this paper, we have assumed that source and channel coding is optimal, quantization is perfect and that a continuum of power levels can be employed. Network lifetime obtained under these assumptions is an upper limit to practically achievable performance. It would be useful to consider the network lifetime problem with more realistic constraints such as fixed coding schemes, finite precision quantizers and a small range of available power levels.

\section{References}

[1] J. Chang and L. Tassiulas, "Energy conserving routing in wireless ad hoc networks," Proc. IEEE INFOCOM 2000, Tel-Aviv, Israel, March 2000.

[2] I. Kang and R. Poovendran, "Maximizing static network lifetime of wireless broadcast adhoc networks," Proc. IEEE ICC 2003, Anchorage, AK, May 2003.

[3] A. Ephremides, "Energy concerns in wireless networks," IEEE Wireless Communications, Vol. 9(4), August 2002.

[4] C. K. Toh, "Maximum battery life routing to support ubiquitous mobile computing in wireless ad hoc networks," IEEE Communications Mag., June 2001.
[5] W. R. Heinzelman, A. Chandrakasan and H. Balalkrishnan, "Energy-efficient communication protocol for wireless microsensor networks," Proc. HICSS 2000, Maui, HI, Jan 2000.

[6] D. Slepian and J. K. Wolf, "Noiseless coding of correlated information sources," IEEE Trans. Inform. Theory, Vol.19, July 1973.

[7] S. S. Pradhan and K. Ramchandran, "Distributed source coding using syndromes: design and construction," Proc. IEEE Data Compression Conference, March 1999.

[8] A. Orlitsky, "Worst-case interactive communication I: Two messages are almost optimal," IEEE Trans. Inform. Theory, Vol.36(5), 1990.

[9] E. Uysal-Biyikoglu, B. Prabhakar and A. El Gamal, "Energy-efficient transmission over a wireless link," IEEE Trans. Networking, Vol.10(4), August 2002.

[10] M. Bhardwaj, A. P. Chandrakasan, "Bounding the lifetime of sensor networks via optimal role assignments," Proc. IEEE INFOCOM 2002, New York City, NY, June 2002.

[11] S. Singh, M. Woo and C. S. Raghavendra, "Power-aware routing in mobile ad hoc networks," Proc. ACM MOBICOM 1998, Dallas, TX, October 1998.

[12] V. Rodoplu and T. Meng, "Minimum energy mobile wireless networks," IEEE JSAC, Vol.17(8), 1999.

[13] N. Sadagopan and B. Krishnamachari, "Maximizing data extraction in energy-limited sensor networks," Proc. IEEE INFOCOM 2004, Hong Kong, March 2004.

[14] Q. Li, J. Aslam and D. Rus, "Online power-aware routing in wireless ad hoc networks," Proc. ACM MOBICOM 2001, Rome, Italy, June 2001.

[15] R. Cristescu, B. B. Lozano and M. Vetterli, "On network correlated data gathering" Proc. IEEE INFOCOM 2004, Hong Kong, March 2004.

[16] S. J. Baek, G. de Veciana and X. Su, "Sensor networks through distributed data compression and hierarchical aggregation" IEEE JSAC Vol.22, August 2004.

[17] T. Cover and J. Thomas, Elements of Information Theory, John Wiley \& Sons, 1991. 